\title{
Design and Optimization of Recycle Policies for Multicomponent Azeotropic Distillation Processes with Bifurcation Analysis
}

\author{
Stefan Brüggemann and Wolfgang Marquardt ${ }^{*}$ \\ Lehrstuhl für Prozesstechnik \\ RWTH Aachen University, Templergraben 55, 52056 Aachen, Germany
}

\begin{abstract}
A novel design method for finding cost-optimized recycle policies for multicomponent azeotropic distillation processes is presented. The design task is formulated in form of a nonlinear optimization problem with the aim of minimizing the energy consumption of the process. The minimum energy demand of each column is determined using the rectification body method (RBM). Closure of the mass balance around each unit of the process is enforced by linear constraints. The feasibility of the split in each column with respect to the distillation boundaries is formulated as a nonlinear constraint. This measure of split feasibility is obtained from a bifurcation analysis of the pinch map which is based on reversible distillation theory. The ternary pressure-swing distillation of an ethanol-water mixture using acetone as an entrainer is used as an illustrative example for the optimization and the assessment of split feasibility.
\end{abstract}

Keywords: Distillation design, nonlinear optimization, recycle policy, split feasibility, bifurcation analysis, rectification body method.

\section{Introduction}

Many azeotropic mixtures exhibit distillation boundaries that limit the reachable products of simple distillation columns (Doherty and Malone, 2001). Such mixtures cannot be separated by a sequential series of columns. Instead, processes in which the columns are coupled by one or more recycle streams must be utilized. Usually there are several competing design alternatives. A typical example is the purification of an ethanol-water mixture where process alternatives based on heterogeneous distillation (Pham et al., 1989, Urdaneta et al., 2002), extractive distillation (Brüggemann and Marquardt, 2003), pressure-swing distillation (Knapp and Doherty, 1992), exploitation of a highly curved boundary (Widagdo and Seider, 1996) and combination of distillation and pervaporation (Bausa and Marquardt, 2000) are known. Due to the recycle streams, the mass balances of each of these processes are characterized by one or more degrees of freedom. In order to rate the economic potential of any of these alternatives in the conceptual design phase the respective degrees of freedom have to be optimized with respect to a suitable cost definition. However, the limitation of the splits

*Author to whom correspondence should be adressed : marquardt@lpt.rwth-aachen.de 
by the distillation boundaries is a major obstacle to the formulation of the optimization model because information about the distillation boundaries is only available implicitly, e.g. as a visualization of the residue curve map. The feasibility of a proposed process is then subject to a graphical analysis which can hardly be automated. Therefore the optimization problem cannot be solved using any standard algorithm. Instead, several authors (Knapp and Doherty, 1992, Knight and Doherty, 1989) propose methods to rank the importance of the optimization variables and then perform several one-dimensional sensitivity analyses in order to find the optimal solution. It can easily be imagined that this is a relatively time-consuming and tedious operation. Due to the limitations of visualization such methods are further limited to ternary mixtures.

In this contribution a novel formulation of the optimization problem is proposed. Concentrations on distillation boundaries are determined from bifurcation analysis on pinch maps. Subsequently, split feasibility can be calculated from an equation system of the form $g(x)=0$ and the optimization model can be solved by standard algorithms eliminating the need for manual intervention of the design engineer. The separation of an ethanol-water mixture in a ternary pressure-swing distillation process will be used as an illustrative example for the new design approach.

\section{Distillation Boundaries and Determination of Split Feasibility}

The set of feasible compositions in a section of a distillation column are bounded by the distillation boundaries. The location and the form of these boundaries depend on two independent parameters, namely the product compositions $\mathbf{x}_{\mathrm{D}}$ and $\mathbf{x}_{\mathrm{B}}$ and the reflux ratio $r$. For conceptual process design, the parametric dependence of these continuous distillation boundaries (CDB, Fidkowski et al., 1993) is quite bothersome because they need to be recalculated at each operating point. Therefore approximations of the real boundaries which eliminate this dependency are usually used for design. To illustrate this approximation the ternary example mixture is introduced first.

\subsection{Illustrative Separation Example}

The ethanol-water mixture is characterized by a binary minimum azeotrope. The location of this azeotrope is highly pressure-dependent as reported in several textbooks. However, the use of this phenomenon for separation of a highly diluted ethanol solution into the two pure components is severely limited by the low driving force close to the azeotrope which leads to frequent formation of tangent pinches. Knapp and Doherty (1992) therefore promote a pressure-swing process alternative operating at pressures of $1 \mathrm{~atm}$ and $10 \mathrm{~atm}$ in which acetone is added as an entrainer. Physical property data and feed composition are taken from their publication. Further, an overall feed flowrate of $F=1 \mathrm{kmol} / \mathrm{s}$ is assumed.

\subsection{Simple Distillation Boundaries (SDB)}

Figure 1 shows a residue curve map for the ternary mixture. At 1 atm the ethanol-water azeotrope is the only azeotrope of the mixture. The corresponding distillation boundary is connected to the pure acetone vertex. At $10 \mathrm{~atm}$ two additional binary azeotropes appear on the other two binary edges. Furthermore, a minimum boiling ternary azeotrope is found. The residue curves connecting the azeotropes and the pure 

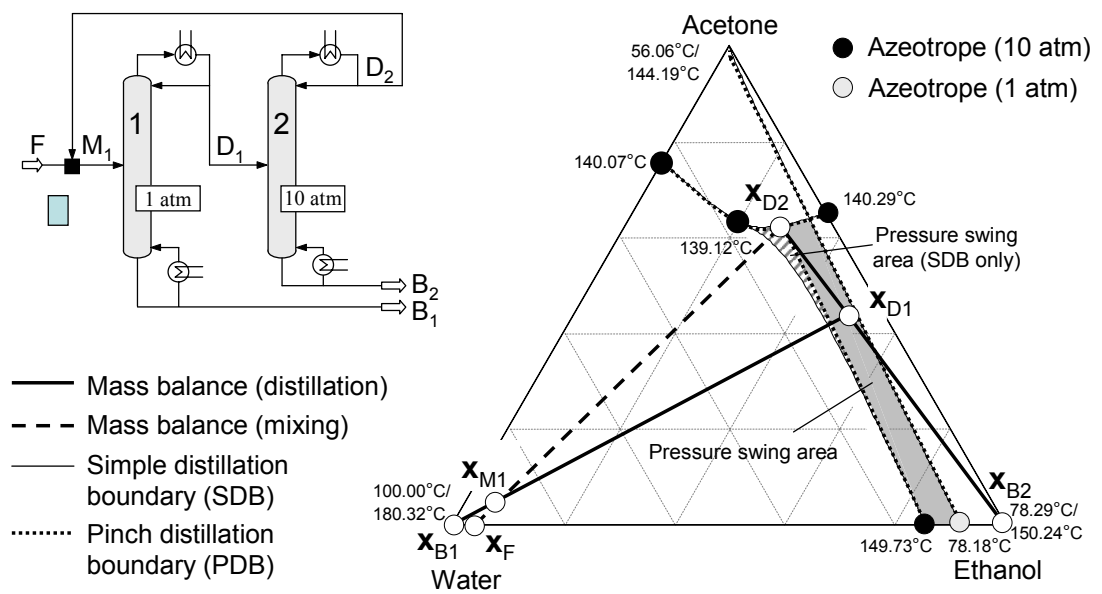

Figure 1: Flowsheet (left) and optimized mass balance of the pressure-swing distillation process in a ternary diagram (right). The ternary diagram also shows the azeotropes at both pressure levels and the simple and pinch distillation boundaries.

component vertices are called simple distillation boundaries (SDB). They limit the feasible product concentrations for columns operating at infinite reflux. For conceptual design, they are often assumed to qualitatively also hold for finite reflux. Using this assumption the shaded and the hatched regions between the distillation boundaries at both pressures marks the region suitable for the pressure swing. However, violation of the SDB by columns with finite reflux has been reported frequently in the literature (Wahnschafft et al., 1992). Another major drawback for the use of SDB in the context of optimization is that points on SDB cannot be by an equation of the form $\mathrm{g}(\mathrm{x})=0$. Instead, the feasibility of a split can only be determined by the calculation of a residue curve starting from the product composition by a set of ordinary differential equations.

\subsection{Pinch Distillation Boundaries (PDB)}

A second approximation of the distillation boundaries can be obtained from reversible distillation theory. In this case concentration profiles are known to be identical to the pinch branches (Koehler et al., 1991). Hence, distillate and bottom product must be connected by a pinch branch. Figure 2 shows the pinch branches for three different choices of the distillate composition $\mathbf{x}_{\mathrm{D}}$. For $x_{\text {D,Water }}<z_{\text {Water }}$, i.e. a distillate composition right of the boundary, a pinch branch connects the distillate with the pure ethanol vertex. For $x_{D, \text { Water }}>z_{\text {Water }}$, i.e. a distillate composition left of the boundary, the distillate is connected to the pure water vertex. In both cases an additional pinch branch exists which is not connected to the distillate composition. For $x_{D \text {, Water }}=z_{\text {Water }}$, i.e. a point on the boundary, the two pinch branches intersect. Hence, both potential bottom products, pure ethanol and pure water, are reachable from this distillate composition. In the context of bifurcation theory, Davydian et al. (1997) relate the intersection of the pinch branches to a pitchfork bifurcation point. Such bifurcation can be located on a pinch branch with 

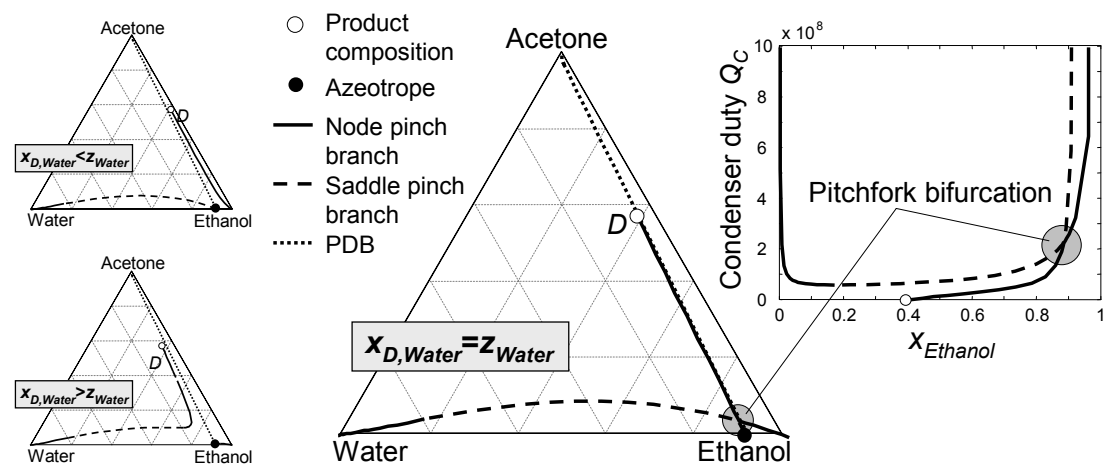

Figure 2: Ternary diagrams of pinch branches for different compositions $x_{D}$. For $x_{D}=z$, i.e. on the distillation boundary, the condenser heat duty $Q_{C}$ is shown as a function of the ethanol concentration along the pinch branches.

$\mathbf{f}_{\mathrm{P}}(\mathbf{s})= \begin{cases}(1-v) x_{i}+v y_{i}-z_{i}=0 & i=1, \ldots, C, \\ y_{i}-K_{i}(\mathbf{x}, \mathbf{y}, T, p)=0 & i=1, \ldots, C, \\ 1-\sum_{k=1}^{C} y_{k}=0 & \end{cases}$

where $\mathbf{z}$ is the product composition by applying the conditions for a stability change

$\sum_{j=1}^{2 C+1} \frac{\partial f_{P, j}}{\partial s_{i}} w_{j}=0$

$$
i=1, \ldots, 2 C+1 \text {, }
$$

$1-\sum_{j=1}^{2 C+1} w_{j}^{0} w_{j}=0$

on the set of pinch equations $\mathbf{f}_{\mathrm{P}}(\mathbf{s})$ with $\mathbf{s}=(\mathbf{x}, \mathbf{y}, T)$, where $\mathbf{w}$ is a null vector and $\mathbf{w}^{\mathbf{0}}$ is a null vector of a previous iteration, together with the pitchfork condition

$\sum_{j=1}^{2 C+1} \frac{\partial f_{P, j}}{\partial v} w_{j}=0$

(Brüggemann and Marquardt, 2003). The locus of the concentrations $\mathbf{z}$ complying with the system of equations presented above is termed pinch distillation boundary (PDB).

\subsection{Assessing Split Feasibility using the Pinch Distillation Boundary}

Inspection of the PDB system of equations above reveals that there are $C$ - 1 degrees of freedom remaining. However, when checking a proposed split given by the distillate and bottom product compositions $\mathbf{x}_{\mathrm{D}}$ and $\mathbf{x}_{\mathrm{B}}$ for feasibility, the mass balance line defines an unique point $\mathbf{z}$ on the boundary (see Figure 3, left). Using the distances $a$ and $b$ a feasibility parameter $m$ can be defined. A feasible split requires $m \geq 1$. However, the necessary solution of $\mathbf{z}$ can only be obtained if a good initialization of $\mathbf{s}$ is known. The saddle azeotrope can be used to provide a starting point $\mathbf{z}^{0}$ on the distillation boundary. The mass balance of the split is then modeled by the homotopy formulation

$z_{i}-\lambda\left((1-m) x_{B, i}+m x_{D, i}\right)-(1-\lambda)\left((1-m) x_{B, i}+m z_{i}^{0}\right)=0 \quad i=1, \ldots, C$. 


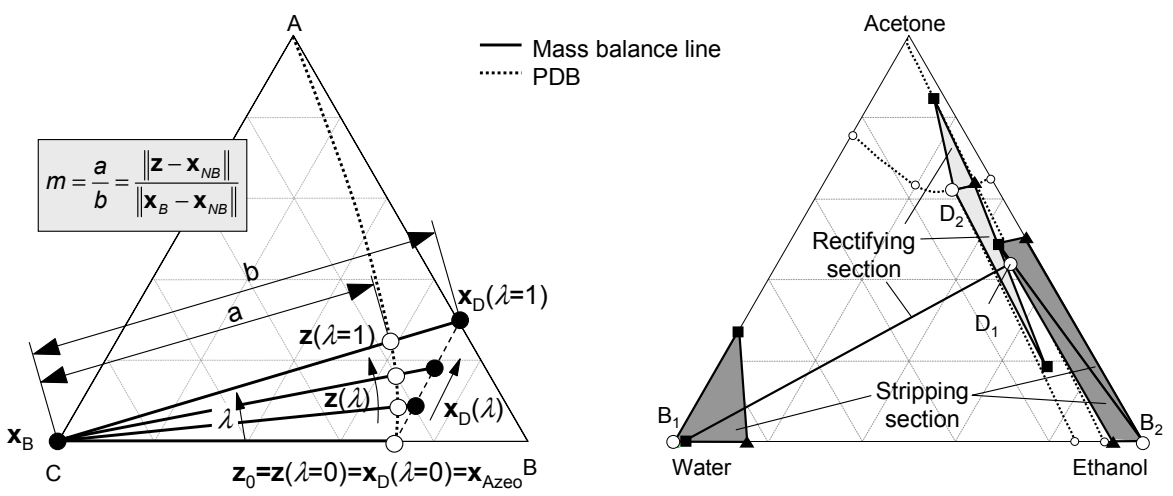

Figure 3: Left: Homotopy continuation for determination of split feasibility. Right: Rectification bodies of both columns at optimal solution.

At $\lambda=0$ the balance line is deformed to run from the bottom product $\mathbf{x}_{\mathrm{B}}$ to the azeotrope (see Figure 3). A solution for eqns. (1)-(6) is easily obtained by initializing all state variables with the values at the azeotrope $\mathbf{s}^{0}=\left(\mathbf{x}=\mathbf{y}=\mathbf{z}=\mathbf{x}_{\text {Azzo }}, T=T_{\text {Azeo }}, v=\infty, \mathbf{w}=\mathbf{w}^{0}\right)$ where $\mathbf{w}^{0}$ is an eigenvector of the azeotrope. Figure 3, left, shows the path of the continuation. Note that if more than one azeotrope exists, continuation runs have to be carried out for all azeotropes. Feasibility is then subject to the smallest positive value of $m$.

\section{Optimization on the Flowsheet Level}

Using the split feasibility parameter $m$ obtained from the homotopy continuation algorithm the optimization of the pressure-swing process can now be automated.

\subsection{Optimization Model and Properties of the Solver}

A general formulation for the minimization of the overall reboiler heat demand of the pressure-swing process can be modeled

$\min f(\mathbf{u})=\sum_{k \in C O L} Q_{B \min , k}(\mathbf{u})$

$\mathbf{A} \mathbf{u}-\mathbf{b}=0$

$m_{k}(\mathbf{u})-1 \geq 0$

$k=1, \ldots, C O L$

$u_{j} \geq 0$

$j=1, \ldots, 3 C$

where $\mathbf{u}$ is the set of all unknown component streams in the process, i.e. $m_{\mathrm{i}}, d_{1, i}, d_{2, i}$ with $i=1, . ., C$ (see Figure 1). Closure of the mass balances is enforced by the linear constraints (8), whereas the nonlinear constraints (9) enforce split feasibility. The minimum reboiler heat demand $Q_{B m i n, k}=f(\mathbf{u})$ of each column $k$ is determined by the rectification body method (RBM, Bausa et al., 1998) which exploits the pinch information in order to construct geometrical bodies that are dependent on the energy supplied to the separation. Application of the RBM algorithm requires that the split is feasible. Therefore, a feasible path optimization algorithm must be used in order to successfully solve the optimization problem. Here, the solver CFSQP (Lawrence and Tits, 2001) has been chosen. 


\subsection{Results for the Pressure-Swing Process}

The optimization converges after 33 iterations. A minimum overall reboiler heat duty of $Q_{B T, \min }=32.31 \mathrm{MW}$ is found where $Q_{B l, \min }=18.90 \mathrm{MW}$ and $Q_{B 2, \text { min }}=13.41 \mathrm{MW}$. Figure 1 gives a visualization of the mass balance in a ternary diagram. Figure 3, right, shows the rectification bodies for both columns. It can be seen that the linear rectification body of the rectifying section of column 1 largely overlaps the corresponding triangular body of the stripping section. This is due to the appearance of a tangent pinch in the rectifying section. For the same separation example, Knapp and Doherty (1992) find slightly lower minimum energy demands of $Q_{B 1, \min }=16.13 \mathrm{MW}$ and $Q_{B 2, \min }=14.49 \mathrm{MW}$ using the boundary value method. The total heat demand of their process is therefore $Q_{B T, m i n}=30.62 \mathrm{MW}(-5.2 \%)$. Their choice of the mass balance $\mathbf{u}$ is qualitatively similar to the one obtained by the RBM optimization. However, their choice of $\mathbf{x}_{\mathbf{D} 2}$ is outside the valid PDR but inside the additional pressure-swing region indicated by the SDR (see Figure 1). The slightly more energy-efficient performance of the Knapp and Doherty process in comparison to the one obtained by the approach presented here can be attributed to this choice of $\mathbf{x}_{\mathbf{D} 2}$.

\section{Conclusions}

A conceptual design method for the separation of azeotropic mixtures with distillation boundaries based on nonlinear optimization has been presented. The method allows for rapid screening of the economic potential and therefore directly supports the selection of a cost-optimal process from a set of process alternatives. The use of bifurcation analysis for the determination of the pinch distillation boundary allows to assess split feasibility automatically eliminating the need for graphical visualization of residue curve maps. The method has been applied to a ternary pressure-swing process here. However, it can in principle be applied to mixtures with an arbitrary number of components.

\section{References}

Bausa, J., R. v. Watzdorf and W. Marquardt, 1998, AIChE J. 44, 2181.

Bausa, J. and W. Marquardt, 2000, Ind. Eng. Chem. Res. 39, 1658.

Brüggemann, S. and W. Marquardt, 2003, Accepted for publication in AIChE J.

Davydian, A. G., M. F. Malone and M. F. Doherty, 1997, Theo. Found. Chem. Eng 31, 327.

Doherty, M. F. and M. F. Malone, 2001, Conceptual Design of Distillation Systems. McGraw-Hill, New Jersey.

Fidkowski, Z. T., M. F. Doherty, M. F. Malone, 1993, AIChE J. 39, 1303.

Knapp, J. P. and M. F. Doherty, 1992, Ind. Eng. Chem. Res. 31, 346.

Knight, J. R. and M. F. Doherty, 1989, Ind. Eng. Chem. Res. 28, 564.

Koehler, J., P. Aguirre and E. Blass, 1991, Chem. Eng. Sci. 46, 3007.

Lawrence, C. T. and A. L. Tits, 2001, SIAM J. Opt. 11, 1092.

Pham, H. N., P. J. Ryan and M. F. Doherty, 1989, AIChE J. 35, 1585.

Urdaneta, R. Y., J. Bausa, S. Brüggemann and W. Marquardt, 2002, Ind. Eng. Chem. Res. 41, 3849.

Wahnschafft, O. M., J. W. Koehler, E. Blass and A. W. Westerberg, 1992, Ind. Eng. Chem. Res. 31, 2345.

Widagdo, S. and W. D. Seider, 1996, AIChE J. 42, 96. 